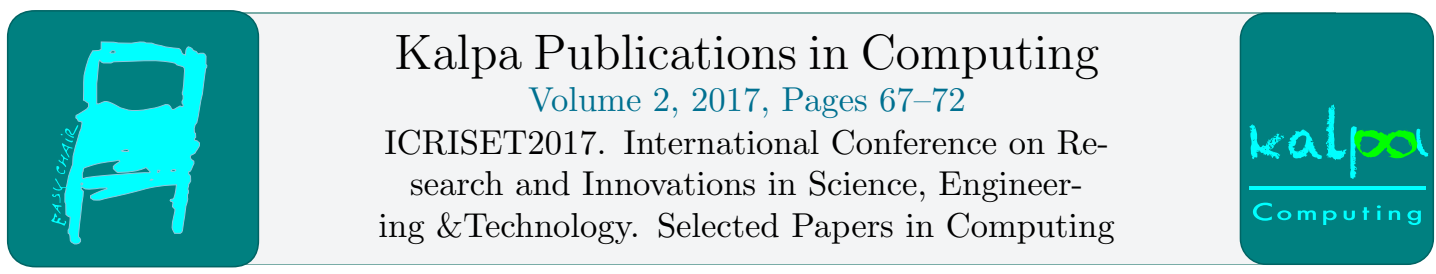

\title{
Indian Currency Recognition using Neural Network Pattern Recognition Tool
}

\author{
Mr.Viranchi N Patel ${ }^{1}$, Dr.Udesang K Jaliya ${ }^{2}$ and Mr.Keyur N Brahmbhatt ${ }^{3}$ \\ ${ }^{1}$ M.tech Computer Engineering, BVM Engineering College, V.V.Nagar, India \\ ${ }^{2}$ Asst. Prof.: Computer Department, BVM Engineering College, V.V.Nagar, India \\ ${ }^{3}$ Asst. Prof.: Information Technology, BVM Engineering College, V.V.Nagar, India \\ viranchi.viranchipatel@gmail.com, udayjaliya@gmail.com, \\ keyur.brahmbhattebvmengineering.ac, in
}

\begin{abstract}
Advancement of Technology has replaced humans in almost every field with machines. By introducing machines, banking automation has reduced human workload. More care is required to handle currency, which is reduced by automation of banking. The identification of the currency value is hard when currency notes are blurry or damaged. Complex designs are included to enhance security of currency. This makes the task of currency recognition very difficult. To correctly recognize a currency it is very significant to choose the good features and suitable algorithm. In proposed method, Canny Edge Detector is used for segmentation and for classification, NN pattern recognition tool is used which gives $95.6 \%$ accuracy.
\end{abstract}

\section{Introduction}

Currency is the paper notes and normal coins, which is releases for circulating within an economy by the government. It is the medium of switch for services and goods. For transaction, paper currency is an important medium. Characteristics of paper currency are simplicity, durability, complete control and cheapness. Due to this, it become popular. Among all other alternative forms of currency, the most preferable form of the currency is the paper. There is a one drawback of paper currencies which is that it cannot be reused but compared with the other methods this problem is not that much serious. As the part of the technological progression introduced to the banking sectors, financial institution and banking had started financial self-services. By using ATM counter and Coin - dispenser automated banking system is achieved where machines are used to handled currencies. In such situations, the machine will use the currency recognizer for the classification of the bank notes. (Vishnu R B. O., Currency Detection Using Similarity Indices Method, April 2014)

Currency has two types of features internal and external features. External features include physical aspects of the currency like width and size. But these physical features are not reliable because currencies may damage due to circulation. Due to this damaged currencies system may fail to 
recognize currencies. Internal feature includes the Color feature, which is also not reliable because currencies are passed through various hand and due to this, it becomes dirty which may give incorrect result. For currencies of each denomination there is a specific color and size followed by Reserve bank of India. (Vishnu R B. O., Currency Detection Using Similarity Indices Method, April 2014) (Vishnu R B. O., Principal component analysis on Indian currency recognition, Sept. 2014)

It is a very simple for human to identify the denomination of currency note because our brain is extremely skillful in learning new matters and discovering them later without much trouble. But this currency recognition task turns very challenging in computer vision, in cases when currencies becomes damaged, old, and faded due to wear and tear. (MrigankaGogoi, Feb. 2015) Security features are included in every Indian Currency which provides help in recognition and identification of the currency value. Various Security features are identification mark (shape), Center value, Ashoka, Latent image, See through register, Security thread, Micro letter, Watermark and RBI seal. (htt)

\section{Related Work}

Vishnu R, Bini Omman (Vishnu R B. O., Currency Detection Using Similarity Indices Method, April 2014) proposed a method where the system compare the features, which are extracted from the input note by computing the similarity measure between the features of the input note and the template image of the corresponding feature. Jaccard Similarity Indices is used for calculating the measured similarly.

MrigankaGogoi, Syed Ejaz Ali, Subra Mukherjee (MrigankaGogoi, Feb. 2015) proposed a method for currency value classification. They used not only one feature but included three features which are Aspect Ratio, Color feature and most important the Shape feature also known as Identification mark feature. They first extract the color feature from the note. Then they take the dimensions of the currency and find the aspect ratio. Next step is the portion in which identification mark is present is segmented. Then by using "Fourier Descriptor", they obtain most important feature, which is the shape of I.D. mark. Once the features are extracted, apply Artificial Neural Network to classify the shapes and on the basis of this classification, values of various Indian Currencies are recognize.

Vishnu R, Bini Omman (Vishnu R B. O., Principal component analysis on Indian currency recognition, Sept. 2014) first proposed a method in which images are normalized by using histogram equalization. Then they extract five features (Shape, Center, RBI seal, Micro Letter, Latent image) from images of currency by placing a rectangular box of specific dimensions which discovers the Region of Interest (ROI). Once the features are extracted PCA (principal component analysis) is applied to each of these features. Finally, the distance between the weight vectors of test image with weight vector of each training images is calculated using Mahalanobis distance method. Then the class of test image is the class of training image for which the similarity is found to be high.

Jayant kumar Nayak, Chaitan Majhi, Apurva Kumar Srivastav, Ajaya Kumar Dash (Jayant kumar Nayak, Dec. 2015) proposed a method for indian currency recognition in which they used the region of interest that is the region containing the face value of the paper currency note. They found the region of interest and its masking using active contours without edges segmentation by Chan Vese algorithm.

Vishnu R, Bini Omman (Vishnu R B. O., Dec. 2014) used PCA (Principal Component Analysis) method and for data Validation WEKA Classifier is used. In this method to generate training model, extracted features in training set is loaded. Based on this training model classifier, decide the class of test feature. Model classification and generation is performed using WEKA. Three classification algorithms such as Random Forest, Support Vector and Naive Bayes used for model prediction and generation. 


\section{Proposed Method}

\subsection{Block Diagram}

Proposed block diagram is shown in Figure1,

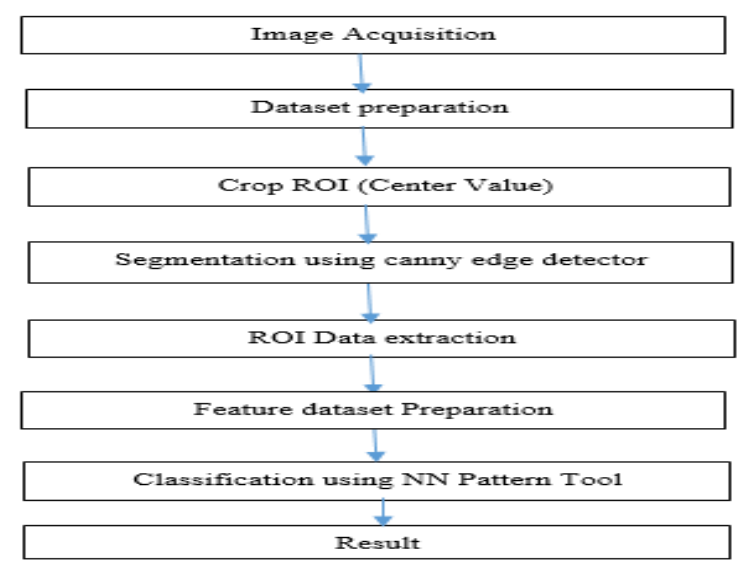

Figure1: Proposed Diagram

\subsection{Image Acquisition}

We have 90 scanned images for each denomination of 5,10,20,50,100,500 paper rupee notes. So in total we have 540 images. Sample scan image is shown in Figure2.

\subsection{Preprocessing}

Following preprocessing step are needed to be applied,

\subsubsection{Rotate and Crop}

Images are scanned in A4 size boundary but we are interested only the in currency part, so first rotate images to 90 degree and then crop the image by setting the dimensions.

\subsubsection{Convert to standard dimensions}

We know that all currencies are different in dimension so to apply uniform logic to all currencies, we first convert all currencies to standard dimension of $250 * 150$.

\subsection{Region of Interest}

We are interested in central numeral value (Face value) of currency. In all denomination of currency this value is located at specific location so, apply single universal ROI to crop the portion of central value. For this we use mask [90 3664 38]. Dimension of Cropped image is 65*39. Which is shown in Figure 3.

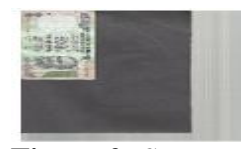

Figure 2: Scan Image

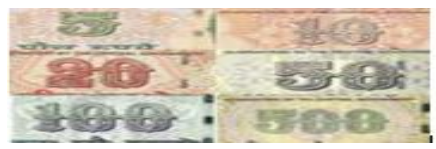

Figure3: Crop ROI 


\subsection{Segmentation}

To get segmented images from crop images following series of steps are performed,

\subsubsection{RGB to gray scale conversion}

RGB images of crop ROI are converted into gray scale images.

\subsubsection{Canny edge detector}

There are various methods for edge detection like Roberts edge detector, Sobel edge detector, Prewitt edge detector and Canny edge detector. Among this Canny is a powerful algorithm because it has low error rate, minimum distance between edge detected by detector and actual edge and only one response to single edge. Canny performs series of step which is [6, 7], Gaussian filter to remove noise, Sobel Operator, Finding Gradient angle, Tracing the edge in the image using theta (angle), Non maximum Suppression, Hysteresis (Two threshold).In proposed methodology used threshold value is 0.15 .

\subsubsection{Morphological operations}

Following morphological operations are performs,

\subsubsection{Open area operation}

This operation removes small objects from binary images which means that objects, which contain lower pixels than specified pixels (P), are removed from the binary image.

\subsubsection{Closing operation}

Closing means dilation operation followed by erosion operation. It is used for eliminating the small holes from the image.

\subsubsection{Dilation operation}

Dilation operation is important morphological operation which adds pixels to the object's boundary in an image.

\subsubsection{Fill operation}

This operation is used to fill hole in images.

\subsubsection{Region Prob Operation}

It is used to measure properties of image regions. First find the connected component using 8 Connectivity which returns the number of objects and pixel list of that object, then find area and eccentricity of object. Next is find label matrix of connected component and finally set appropriate area and eccentricity. Figure 4 shows segmented image.

\subsection{Data Extraction}

To get final extracted images first convert the cropped ROI images to gray scale images and segmented images to binary images by dividing it to 255 . Finally multiply both the images to get final extracted images. Figure 5 shows extracted images. 


\subsection{Laplacian Filter}

Laplacian filter is used for sharpening the image. By implementing the Laplacian filter we get a new image that highlights the edges and other discontinuities of an image. Filter image is shown in Figure6. Four neighbourhood Laplacian filter can be given by [9],

$$
\nabla^{2} \mathrm{f}=[\mathrm{f}(\mathrm{x}+1, \mathrm{y})+\mathrm{f}(\mathrm{x}-1, \mathrm{y})+\mathrm{f}(\mathrm{x}, \mathrm{y}+1)+\mathrm{f}(\mathrm{x}, \mathrm{y}-1)]-4 \mathrm{f}(\mathrm{x}, \mathrm{y})
$$

Mask can be given by,

\begin{tabular}{|r|r|r|}
\hline $\mathbf{0}$ & $\mathbf{1}$ & $\mathbf{0}$ \\
\hline $\mathbf{1}$ & $-\mathbf{4}$ & $\mathbf{1}$ \\
\hline $\mathbf{0}$ & $\mathbf{1}$ & $\mathbf{0}$ \\
\hline
\end{tabular}

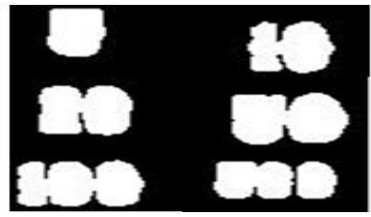

Figure4: Segmented Image

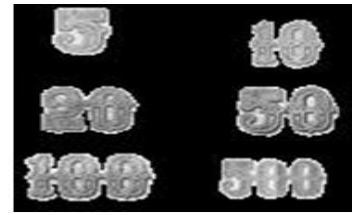

Figure5: Extracted Image

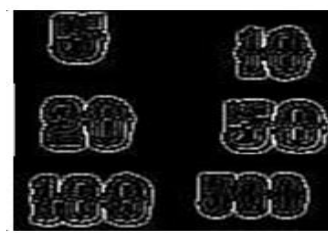

Figure6: Filter Image

\subsection{Database Preparation}

Database contains two variable $\mathrm{X}$ and $\mathrm{Y}$. Here we make pixel-based database. Variable $\mathrm{X}$ contains images verses pixels matrix. We have 540 images so total 540 row in variable $\mathrm{X}$ and dimension of each images is $65^{*} 39$. So total pixels are 2535 and so total 2535 rows in $X$. Therefore, $X$ is a matrix of $540 * 2535$ size. Variable $\mathrm{Y}$ contains images verses class of image matrix. We have total six types of currencies so $\mathrm{Y}$ is a matrix of $540 * 6$ size.

\section{Classification and Result}

For classification Neural Network Pattern Recognition app is used which is available in MATLAB. Start by typing nnstart in command window of MATLAB. The Neural Network Pattern Recognition app will help you to train a network of selected data, and measure its performance using cross-entropy and confusion matrix. It is a two layer feed forward network. It contains sigmoid hidden. After starting you need to load input and target. So we load X and Y variable. Next step is select percentage of training, Validation and testing data. Then select number of hidden layer. Finally train the network.

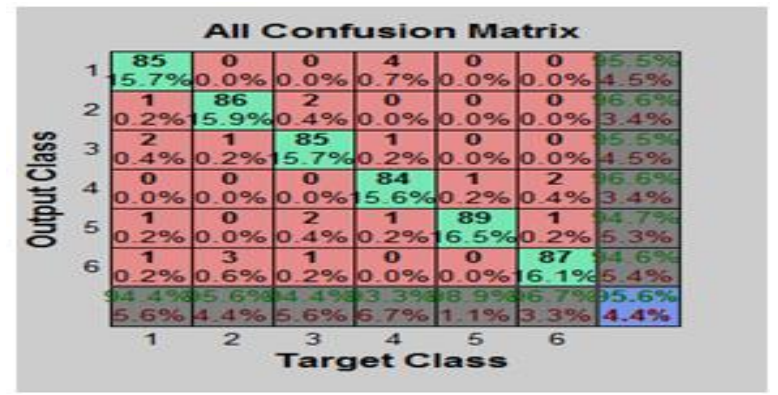

Figure7: All confusion matrix 
Results are shown in following tables,

\begin{tabular}{|c|c|c|c|c|}
\hline $\begin{array}{c}\text { Curr } \\
\text { ency }\end{array}$ & $\begin{array}{c}\text { Total } \\
\text { images }\end{array}$ & $\begin{array}{c}\text { Correctl } \\
\mathbf{y} \\
\text { classifie } \\
\mathbf{d}\end{array}$ & $\begin{array}{c}\text { Incorr } \\
\text { ectly } \\
\text { classifi } \\
\text { ed }\end{array}$ & $\begin{array}{c}\text { Recog } \\
\text { ni- } \\
\text { tion } \\
\text { rate\% }\end{array}$ \\
\hline 10 & 90 & 85 & 5 & 94.4 \\
\hline 20 & 90 & 86 & 4 & 95.6 \\
\hline 50 & 90 & 85 & 5 & 94.4 \\
\hline 100 & 90 & 84 & 6 & 93.3 \\
\hline 500 & 90 & 89 & 1 & 98.9 \\
\hline 5 & 90 & 87 & 3 & 96.7 \\
\hline Total & 540 & 516 & 24 & 95.6 \\
\hline
\end{tabular}

\begin{tabular}{|c|c|c|c|}
\hline $\begin{array}{c}\text { Currency } \\
\text { class }\end{array}$ & $\begin{array}{c}\text { Precisio } \\
\mathbf{n} \\
(\boldsymbol{\%})\end{array}$ & $\begin{array}{c}\text { Recall/Sensi } \\
\text { tivity } \\
(\boldsymbol{\%})\end{array}$ & $\begin{array}{c}\text { Specificity } \\
(\boldsymbol{\%})\end{array}$ \\
\hline $1(10 \mathrm{rs})$ & 95.50 & 94.44 & 99.11 \\
\hline $2(20 \mathrm{rs})$ & 96.66 & 95.55 & 99.33 \\
\hline $3(50 \mathrm{rs})$ & 95.50 & 94.44 & 99.11 \\
\hline $4(100 \mathrm{rs})$ & 96.55 & 93.33 & 99.33 \\
\hline $5(500 \mathrm{rs})$ & 94.68 & 98.88 & 98.88 \\
\hline $6(5 \mathrm{rs})$ & 94.56 & 96.66 & 98.88 \\
\hline
\end{tabular}

Table 1: Result

Table 2: Assessment parameters

\section{Conclusion}

This paper describes the technique of currency recognition using Neural Network Pattern Recognition app. Experiment is conducted over 540 images. From all images, central face value is extracted using canny edge detector method and database is prepared. Finally, database is trained using NN pattern recognition app, which gives $95.6 \%$ classification accuracy.

\section{References}

(n.d.). Retrieved from https://www.rbi.org.in/SCRIPTs/ic_banknotessecurity.aspx

Gonzalez, R. C. (n.d.). Digital image processing.

Jayant kumar Nayak, C. M. (Dec. 2015). Neural network approach for Indian currency recognition. Annual IEEE India Conference (INDICON) (p. 6). New Delhi: IEEE.

MrigankaGogoi, S. E. (Feb. 2015). Automatic Indian Currency Denomination Recognition System based on Artificial. 2nd International Conference on Signal Processing and Integrated Networks (SPIN) (p. 6). Noida: IEEE.

Raman Maini, H. A. (2009). Study and comparison of various image edge detection techniques. International Journal of Image Processing (IJIP), 12.

Syed Irfanullah, A. A. (n.d.). Canny Edge Detection Report.

Vishnu R, B. O. (April 2014). Currency Detection Using Similarity Indices Method. International Conference for Convergence of Technology (I2CT) (p. 8). Pune: IEEE.

Vishnu R, B. O. (Dec. 2014). Principal Features for Indian Currency Recognition. Annual IEEE India Conference (INDICON) (p. 8). Pune: IEEE.

Vishnu R, B. O. (Sept. 2014). Principal component analysis on Indian currency recognition. International Conference on Computer and Communication Technology (p. 6). Allahabad: IEEE. 